\title{
Expression, Purification, Refolding, and Characterization of a Neverland Protein From Caenorhabditis elegans
}

\author{
Shuhong Mao ${ }^{1,2,3,4}$, Zhan Song ${ }^{3}$, Mian Wu ${ }^{3}$, Xiaorui Wang ${ }^{3}$, Fuping $L^{1,2,3,4 *}$ and \\ Hui-Min Qin ${ }^{1,2,3,4 *}$ \\ ${ }^{1}$ Key Laboratory of Industrial Fermentation Microbiology, Ministry of Education, Tianjin University of Science and Technology, \\ Tianjin, China, ${ }^{2}$ Tianjin Key Laboratory of Industrial Microbiology, Tianjin University of Science and Technology, Tianjin, China, \\ ${ }^{3}$ College of Biotechnology, Tianjin University of Science and Technology, Tianjin, China, ${ }^{4}$ National Engineering Laboratory \\ for Industrial Enzymes, Tianjin, China
}

\section{OPEN ACCESS}

Edited by:

Eduardo Jacob-Lopes, Federal University of Santa Maria,

Brazil

Reviewed by:

Xiaogiang Ma,

Singapore-MIT Alliance for Research and Technology (SMART), Singapore

Dirk Tischler,

Ruhr University Bochum, Germany

*Correspondence:

Fuping Lu

Ifp@tust.edu.cn; fupinglu2016@sina.com

Hui-Min Qin

huiminqin@tust.edu.cn

Specialty section:

This article was submitted to

Bioprocess Engineering,

a section of the journal

Frontiers in Bioengineering and

Biotechnology

Received: 09 August 2020

Accepted: 24 September 2020

Published: 21 October 2020

Citation:

Mao S, Song Z, Wu M, Wang X Lu F and Qin H-M (2020) Expression,

Purification, Refolding,

and Characterization of a Neverland

Protein From Caenorhabditis elegans. Front. Bioeng. Biotechnol. 8:593041. doi: 10.3389/fbioe.2020.593041
Steroid hormones that serve as vital compounds are necessary for the development and metabolism of a variety of organisms. The neverland (NVD) family genes encode the conserved Rieske-type oxygenases, which are accountable for the dehydrogenation during the synthesis and regulation of steroid hormones. However, the His-tagged NVD protein from Caenorhabditis elegans expresses as inclusion bodies in Escherichia coli BL21 (DE3). This bottleneck can be solved through refolding by urea or the introduction of a maltose-binding protein (MBP) tag at the $N$-terminus. Through further research on purification after the introduction of a MBP tag at the $N$-terminus, the CD measurement and fluorescence-based thermal shift assay indicated that MBP was favorable for the NVD proteins' solubility and stability, which may be beneficial for the large-scale manufacture of NVD protein for further research. The structural model contained the Rieske [2Fe-2S] domain and non-heme iron-binding motif, which were similar to 3-ketosteroid $9 \alpha$-hydroxylase.

Keywords: neverland, maltose-binding protein, refolding, soluble expression, structural model-3-

\section{INTRODUCTION}

Sterol derivatives mediate a wide range of growth, development, and evolution in most living species (Thummel and Chory, 2002). In insects, the steroid hormone ecdysone plays an essential role in the developmental transitions and egg production (Huang et al., 2008). The flies could not reach the adult stage when the synthesis for lathosterol was disabled by shutting down the NVD gene using the RNA interference (RNAi) in vivo. This matter can be solved by supplementing the standard food or lathosterol on time (Lang et al., 2012). The sterol metabolites also had many important properties, mostly related to the biosynthesis and regulation of amino acids and vitamins (Romero et al., 2005), which are involved in cholesterol homeostasis and synthesis of vitamin $\mathrm{D}_{3}$.

The metabolites of vitamin $\mathrm{D}_{3}$ (cholecalciferol) have raised a great concern due to its biological effects and physiological properties, such as calcium metabolism and phosphate homeostasis, regulation of immune responses, promotion of insulin secretion, and stimulation of cell proliferation and differentiation (Di Rosa et al., 2011). The vitamin $\mathrm{D}_{3}$ was synthesized in humans, and most of the vertebrate animals on the skin, in which the 7-dehydroxycholesterol (7-DHC) was converted into pre-vitamin $\mathrm{D}_{3}$ via ultraviolet (UV) irradiation at wavelengths of 290$320 \mathrm{~nm}$ and, meanwhile, followed by a thermal isomerization to form vitamin $\mathrm{D}_{3}$ spontaneously (Tripkovic et al., 2012). The high incidence of renal bone disease, osteomalacia and osteoporosis, 
was reported to be associated with the malabsorption of calcium, which is caused by a deficiency of vitamin $\mathrm{D}_{3}$ (Kanis, 1999). Compared with the chemosynthesis of 7-DHC using cholesterol (Dugas and Brunel, 2006), the bioconversion of cholesterol into 7-DHC has attracted more attention with regioselectivity and no pollution for the environment (Yoshiyama et al., 2006; Wollam et al., 2011; YoshiyamaYanagawa et al., 2011; Lang et al., 2012). The reaction can be catalyzed by the evolutionarily conserved Rieske-domain oxygenase neverland (NVD), which contains a Rieske [2Fe2S] cluster binding domain to function as an electron acceptor and electron transfer, and a highly conserved non-heme iron-binding center as a catalytic domain (Yoshiyama et al., 2006). Several Rieske-domain oxygenase genes from reptiles, insects, nematodes, and deuterostome species had been reported including Anolis carolinensis (protein ID XP_003230725.2), Anopheles gambiae (protein ID EAA04927.5) (Holt et al., 2002), Bombyx mori (protein ID BAE94192.1) (Yamanaka et al., 2005), Caenorhabditis elegans (protein ID CAA98235.2) (Rottiers et al., 2006), Ciona intestinalis (protein ID BAK39961.1) (YoshiyamaYanagawa et al., 2011), Drosophila melanogaster (protein ID ABW08586.1) (Warren et al., 2002), Danaus plexippus (protein ID OWR46621.1) (Zhan et al., 2011), Danio rerio (protein ID BAK39960.1) (Yoshiyama et al., 2006), Gallus gallus (protein ID XP_425346.2) (Yoshiyama et al., 2006), Hemicentrotus pulcherrimus (protein ID BAK39963.1) (Yoshiyama-Yanagawa et al., 2011), Rhodococcus rhodochrous ( $k \operatorname{sh} A$, protein ID ADY18310.1) (Petrusma et al., 2011), Spodoptera littoralis (protein ID ADK56283.1) (Iga et al., 2013), Pseudomonas fluorescens (prnD, protein ID AAB97507.1) (Lee et al., 2005), Podarcis muralis (protein ID XP_028576239.1), Pseudonaja textilis (protein ID XP_026568371.1), and Xenopus laevis (protein ID BAK39959.1) (Yoshiyama-Yanagawa et al., 2011). The family proteins are an essential regulator of cholesterol metabolism and steroidogenesis.

In addition, NVD from Caenorhabditis elegans (CeNVD) were identified in the metabolic pathway of cholesterol, and genetic evidence has demonstrated that the NVD gene plays a vital role in the larval development and adult aging in the ecdysteroid biosynthesis (Rottiers et al., 2006; YoshiyamaYanagawa et al., 2011). However, there have been a few reports about the effective heterologous expression and production system of the NVD family proteins in vitro. Here, we verified that the NVD protein was expressed as inclusion bodies with His-tag (Zhu et al., 2019b), and a small amount of soluble protein was obtained, even though it was further refolded by urea. Subsequently, we introduced the maltose-binding protein (MBP) to enhance the soluble expression and purification of CeNVD in Escherichia coli BL21 (DE3), and the thermostability of CeNVD was also improved.

\section{MATERIALS AND METHODS}

\section{Materials}

The neverland gene from Caenorhabditis elegans (CeNVD) was chemically synthesized in pET-28a(+) (Novagen, Madison, WI,
United States) vector by GENEWZ (Suzhou, China) after codon was optimized. The DNA fragment of 1,110 bp was PCR amplified using gene-specific primers, which contain the EcoRI and HindIII restriction sites at the $5^{\prime}$ - and $3^{\prime}$-terminal, and was cloned into the pMal-c2X (New England Biolabs, Beverly, MA, United States) plasmid vector, which contains an N-terminal MBP-tag and sequence. The E. coli BL21 (DE3) (Novagen, Darmstadt, Germany) strain was employed as a heterologous expression host.

\section{Expression and Purification}

The recombinant plasmid was transformed into an E. coli BL21 (DE3) strain and grown in a Luria-Bertani (LB) medium supplemented with kanamycin $(50 \mu \mathrm{g} / \mathrm{ml})$ or ampicillin $(100 \mu \mathrm{g} / \mathrm{ml})$ with a shaking of $220 \mathrm{rpm}$ at $37^{\circ} \mathrm{C}$. When the optical density at $600 \mathrm{~nm}\left(\mathrm{OD}_{600}\right)$ reached $0.6-0.8,0.5 \mathrm{mM}$, isopropyl- $\beta$-D-thiogalactopyranoside (IPTG) was added to the culture, and the recombinant cells were cultivated with a shaking of $160 \mathrm{rpm}$ at $16^{\circ} \mathrm{C}$ for $16-18 \mathrm{~h}$ to induce the protein expression. After cultivation, the recombinant cells were harvested by centrifugation at 5,000 $\times \mathrm{g}$ for $15 \mathrm{~min}$ at $4^{\circ} \mathrm{C}$ and washed twice with PBS (pH 8.0) (Sun et al., 2019).

In order to purify the CeNVD_pET-28a(+), the washed cells were resuspended in a 30-ml lysis buffer A $(20 \mathrm{mM}$ Tris- $\mathrm{HCl}, 20 \mathrm{mM}$ imidazole, $500 \mathrm{mM} \mathrm{NaCl}$, and $1 \mathrm{mM}$ dithiothreitol, $\mathrm{pH} 8.0$ ) containing $0.5 \mathrm{mg} / \mathrm{ml}$ lysozyme and $1 \mathrm{mM}$ phenylmethanesulfonyl fluoride (PMSF) and disrupted using a sonicator (Sonic Dismembrator Model 100, Pittsburgh, PA, United States) on ice bath for $20 \mathrm{~min}$, the unbroken cells and cell debris were removed by centrifugation at 20,000 $\times g$ for $30 \mathrm{~min}$ at $4^{\circ} \mathrm{C}$, the supernatant was applied to a nickelnitrilotriacetic acid (Ni-NTA) agarose affinity chromatography matrix (QIAGEN, Hilden, Germany), and pre-equilibrated with lysis buffer A. After washing the open column with 10-ml of lysis buffer A extensively, the bound protein was eluted with a $10-\mathrm{ml}$ elution buffer A $(20 \mathrm{mM}$ Tris- $\mathrm{HCl}, 300 \mathrm{mM}$ imidazole, $300 \mathrm{mM} \mathrm{NaCl}$, and $1 \mathrm{mM}$ dithiothreitol, $\mathrm{pH}$ 8.0) (Mao et al., 2018).

For purification of the CeNVD_pMal-c2X, the amylose resin was applied to the fixed MBP_CeNVD fusion protein. The unbound protein was washed with lysis buffer B (20 mM Tris$\mathrm{HCl}, 500 \mathrm{mM} \mathrm{NaCl}, 1 \mathrm{mM}$ EDTA, and $1 \mathrm{mM}$ dithiothreitol, $\mathrm{pH}$ 8.0 ), and the target protein was eluted with $10 \mathrm{ml}$ of elution buffer B (20 mM Tris-HCl, $20 \mathrm{mM}$ maltose, $500 \mathrm{mM} \mathrm{NaCl}, 1 \mathrm{mM}$ EDTA, and $1 \mathrm{mM}$ dithiothreitol, $\mathrm{pH}$ 8.0) (Zhu et al., 2019b). Then the protein was further purified by an anion exchange chromatography employing a Resource Q column (column volume: $6 \mathrm{ml}$, flow rate: $4 \mathrm{ml} / \mathrm{min}$, GE Healthcare, Stockholm, Sweden) on the ÄKTA system (GE Healthcare, Sweden) (Sun et al., 2018). The purified enzyme was eluted with a linear gradient between 0 and $1 \mathrm{M} \mathrm{NaCl}$ at a flow rate of $3 \mathrm{ml} / \mathrm{min}$. Subsequently, the MBP tag was digested using a Factor Xa Protease (New England Biolabs, Beverly, MA, United States) at $4^{\circ} \mathrm{C}$ for $12 \mathrm{~h}$ and then loaded on an amylose resin to remove the MBP tag and undigested protein. The flow-through buffer containing the target protein was collected and concentrated for further experiments. 


\section{Western Blot Analysis of CeNVD_pET-28a(+)}

After the centrifugation of the disrupted CeNVD_pET-28a(+), the cleared supernatant and precipitant were loaded on SDSPAGE gels, then all the protein molecules was transferred to a PVDF membrane and blocked in a PBST buffer (PBS pH 8.0, $0.02 \%$ Tween-20) containing a $1 \%$ bovine serum albumin (BSA) for $2 \mathrm{~h}$, followed by incubation in an anti-His-tag mouse monoclonal antibody (Abcam, Cambridge, United Kingdom), which was diluted in a blocking buffer (PBST pH 8.0, 1\% BSA) at the indicated concentrations of $1: 5,000$ for $12 \mathrm{~h}$ at $4^{\circ} \mathrm{C}$. After washing with PBST for four times, the membrane was protected from light and incubated with the HRP-conjugated secondary antibody (HRP-conjugated goat anti-mouse IgG, Tiangen Biochemical Technology, Beijing, China) at a dilution of 1:1,000 and room temperature for $2 \mathrm{~h}$. After washing, the target protein was trapped by the HRP-DAB chromogenic substrate kit (Tiangen Biochemical Technology, Beijing, China), and the immunoreactive band was digitally scanned using an Odyssey Infrared Imager (LI-COR Bio-science, Lincoln, NE, United States) (Wan et al., 2016).

\section{The Refolding of Denatured Protein}

The CeNVD_pET-28a $(+)$ cells were collected and resuspended in lysis buffer C (20 mM Tris- $\mathrm{HCl}, 20 \mathrm{mM}$ imidazole, $500 \mathrm{mM}$ $\mathrm{NaCl}, 8 \mathrm{M}$ urea, and $1 \mathrm{mM} \mathrm{DTT}, \mathrm{pH} 8.0$ ), and then disrupted and centrifuged as mentioned above. The cleared supernatant containing the denatured enzyme was refolded by sequential dialysis with a gradient descent of urea concentrations $(7,6,5$, $4,3,2,1,0.5,0 \mathrm{M})$, then trapped on a pre-equilibrated Ni-NTA superflow resin (QIAGEN, Hilden, Germany), and washed with lysis buffer D (20 mM Tris- $\mathrm{HCl}, 20 \mathrm{mM}$ imidazole, $500 \mathrm{mM}$ $\mathrm{NaCl}, 1 \mathrm{mM}$ dithiothreitol, $0.5 \mathrm{mM}$ GSSG, $3 \mathrm{mM} \mathrm{GSH}$, and $500 \mathrm{mM}$ arginine, $\mathrm{pH}$ 8.0). The refolded protein was eluted with elution buffer D (20 mM Tris-HCl, $300 \mathrm{mM}$ imidazole, $300 \mathrm{mM}$ $\mathrm{NaCl}, 0.5 \mathrm{mM}$ GSSG, $3 \mathrm{mM}$ GSH, $500 \mathrm{mM}$ arginine, and $1 \mathrm{mM}$ dithiothreitol, pH 8.0) (Qin et al., 2017a). The protein concentration of each purification step was measured via the BCA protein assay kit (Solarbio, Beijing, China) (Qin et al., 2017b).

\section{Molecular Mass Determination}

The molecular weight of the native CeNVD was measured by a gel filtration chromatography using a Superdex200 Increase 10/300 GL column on the ÄKTA system (GE Healthcare, Sweden) (Zhu et al., 2019a). The target enzyme was eluted with a buffer (20 mM Tris-HCl, $150 \mathrm{mM} \mathrm{NaCl}$, and $1 \mathrm{mM} \mathrm{DTT}, \mathrm{pH} \mathrm{8.0)}$ at a flow rate of $1 \mathrm{ml} / \mathrm{min}$ with aldolase $(158 \mathrm{kDa})$, conalbumin $(75 \mathrm{kDa})$ as calibration proteins (GE Healthcare).

\section{Circular Dichroism Measurements}

The circular dichroism (CD) spectra was determined using a MOS-450 CD spectropolarimeter (Biologic, Claix, Charente, France). The protein sample was loaded into a $1-\mathrm{cm}$ path-length quartz cuvette in which $0.1 \mathrm{mg} / \mathrm{ml}$ of protein was dissolved in PBS ( $\mathrm{pH}$ 8.0), and the $\mathrm{CD}$ data were recorded in the far-UV band of $190-250 \mathrm{~nm}$ at room temperature for an average of four times scan with a rate of $1 \mathrm{~nm} / \mathrm{s}$, a bandwidth of $0.1 \mathrm{~nm}$, and a step resolution of $0.1 \mathrm{~nm}$ (Zhu et al., 2019c). Analysis of the protein secondary structure was performed with the program BeStSel ${ }^{1}$ (Micsonai et al., 2015, 2018).

\section{Fluorescence-Based Thermal Shift Assay}

The thermal stability of CeNVD was characterized via the fluorescence-based thermal shift assay using a 48-well assay plate real-time PCR instrument (Bio-Rad, Hercules, CA, United States). Reaction samples were conducted in three replicates that contained $0.4 \mathrm{mg} / \mathrm{ml}$ of protein and $100 \times$ SYPRO Orange dye in PBS buffer $(\mathrm{pH}$ 8.0). The temperature was increased with a linear gradient of $20-90^{\circ} \mathrm{C}$ at $0.5^{\circ} \mathrm{C} / 30 \mathrm{~s}$, and the minimal value was regarded as the melting temperature $\left(T_{m}\right)$ (Mao et al., 2020a).

\section{Structure Modeling of CeNVD}

The three-dimensional (3D) homology model of CeNVD was generated by the SWISS-MODEL (swissmodel.expasy.org/) (Guex and Peitsch, 1997) using a template of 3-ketosteroid 9 $\alpha$-hydroxylases from $R$. rhodochrous (PDB ID: 4QDF, $2.43 \AA$ ) (Penfield et al., 2014), which shared a 30\% sequence identity with CeNVD. Then the generated models were visualized and analyzed using the PyMoL software ${ }^{2}$.

\section{RESULTS AND DISCUSSION}

\section{Sequence Alignment and Phylogenetic Analysis}

The phylogenetic tree of Rieske oxygenase from various microorganisms revealed that the evolutionary relationship of CeNVD was similar to that of C. intestinalis (Figure 1A) with $41.8 \%$ amino acid sequence identity. It showed the lower sequence identity of $30.2 \%$ with $B$. mori. BLAST and sequence analysis indicated that the NVD from $C$. elegans shared a higher sequence identity with $X$. laevis $(45.7 \%), D$. rerio $(44.9 \%)$, G. gallus (44.0\%), C. intestinalis (41.8\%), and A. gambiae $(40.3 \%)$. Amino acid sequence alignment and analysis with the homologous proteins displayed that the family proteins contained two evolutionally conserved domains, Rieske [2Fe2S] domain (C-X-H-X16-17-C-X2-H) and non-heme ironbinding motif [ $\mathrm{Fe}(\mathrm{II})$; E/D-X3-D-X2-H-X4-H]. The conserved residues in CeNVD contained C122, H124, C143, H146 in the Rieske domain, and E230, D234, H237, H242 in the Fe (II) domain (Figure 1B).

\section{Heterologous Expression and Purification of CeNVD Recombinant Enzyme}

The CeNVD_pET28a(+) was expressed in E. coli BL21 (DE3) and purified by the His-trap affinity chromatography. SDS-PAGE

\footnotetext{
${ }^{1}$ http://bestsel.elte.hu

${ }^{2} \mathrm{http}: / /$ www.pymol.org
} 


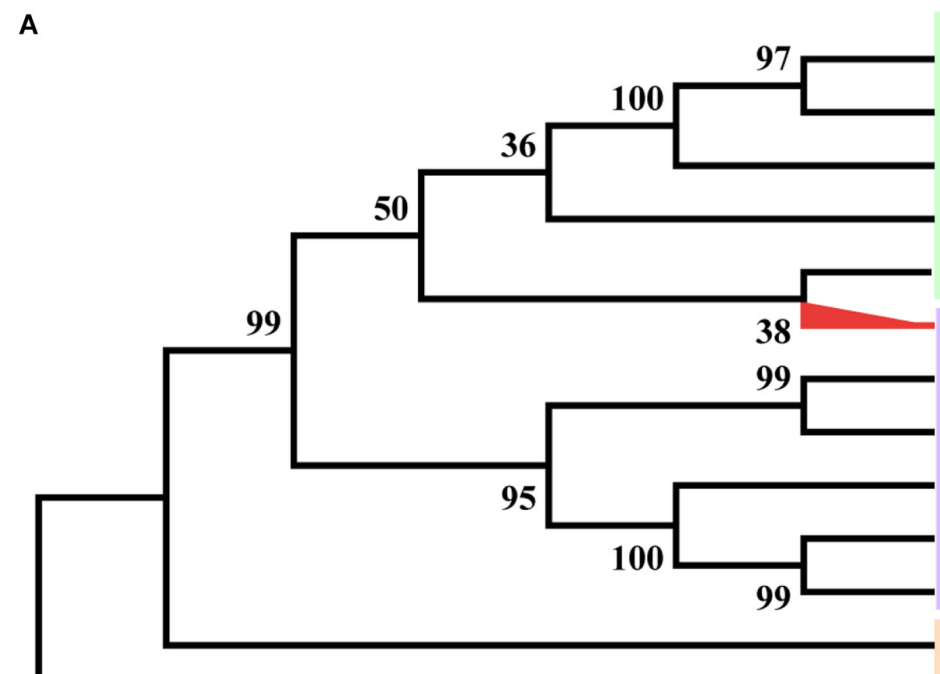

D. rerio

X. laevis

G. gallus

H. pulcherrimus

C. intestinalis

C. elegans

A. gambiae

D. melanogaster

D. plexippus

B. mori

S. littoralis

R. rhodochrous P. fluorescens

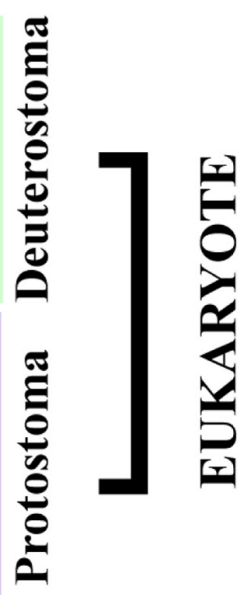

\section{PROKARYOTE}

B

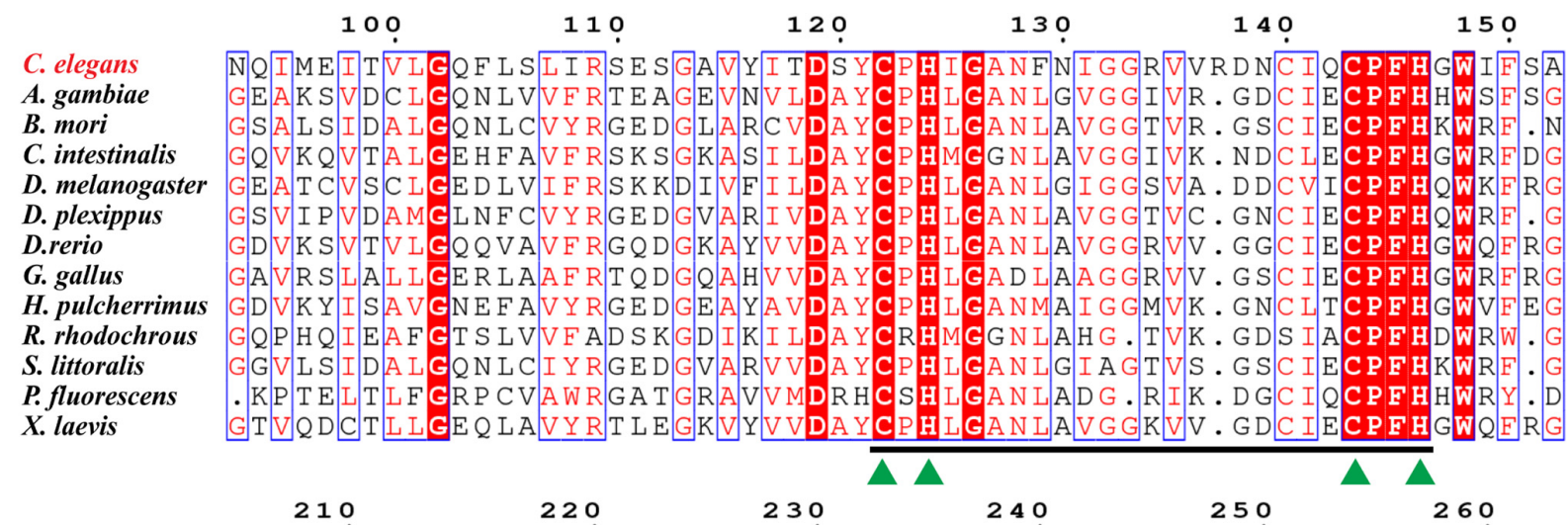

C. elegans

A. gambiae E I T D G F W

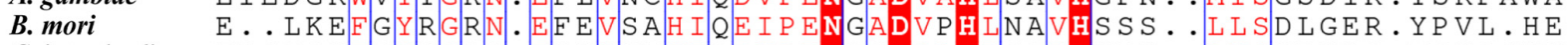

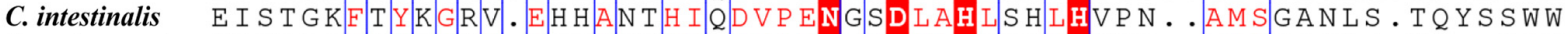

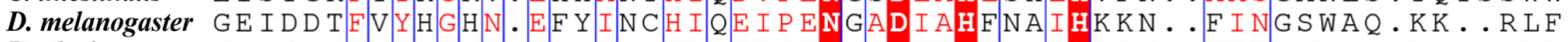

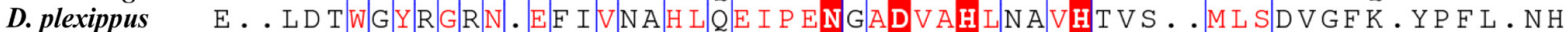

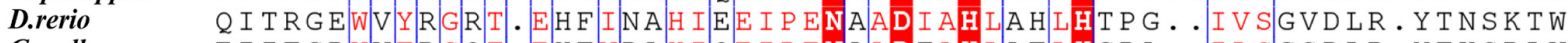

G. gallus $\quad$ I I T G DWV

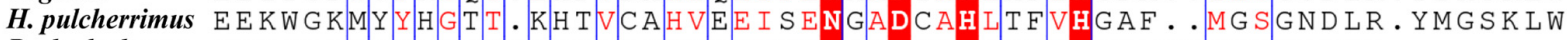

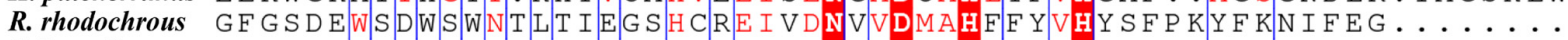

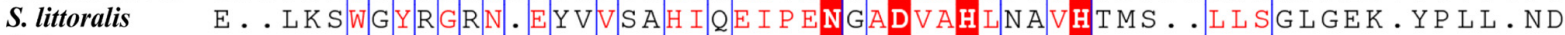

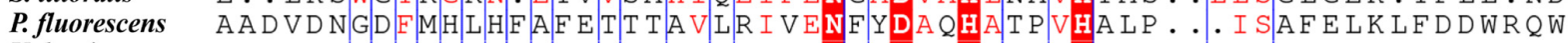

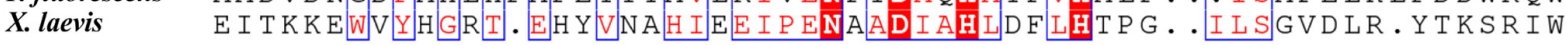

FIGURE 1 | Sequence alignment and phylogenetic tree of Caenorhabditis elegans (CeNVD) with family enzymes. (A) The phylogenetic analysis of Rieske oxygenases from different species. (B) Multiple alignment of CeNVD with other Rieske oxygenases; the green triangle $(\boldsymbol{\Delta})$ and orange asterisk $(\star)$ were responsible for the Rieske [2Fe-2S] domain (C-X-H-X16-17-C-X2-H) and non-heme iron-binding motif [Fe(II); E/D-X3-D-X2-H-X4-H], respectively. The alignment was prepared using the program ESPript 3.0 service (http://espript.ibcp.fr/ESPript/ESPript/).

and Western blot analysis demonstrated that the target protein appeared as a single band with a molecular mass of approximately $42 \mathrm{kDa}$, consistent with the calculated molecular weight of 42,800 Da. However, the protein was overwhelmingly expressed as inclusion bodies (Figures 2A,B). Subsequently, the CeNVD gene was cloned and inserted into pMal-c2X. The reconstructed enzyme was overexpressed and purified by a genericmultiple-step purification using an amylose fast flow resin and anion exchange chromatography (Figures 3A,B). The MBP-tag was then digested by a Factor $\mathrm{Xa}$ protease and removed by an amylose resin (Figure 3C). The yields and purities of CeNVD for the different purification stages are summarized in Table 1. Finally, $4.1 \mathrm{mg}$ 

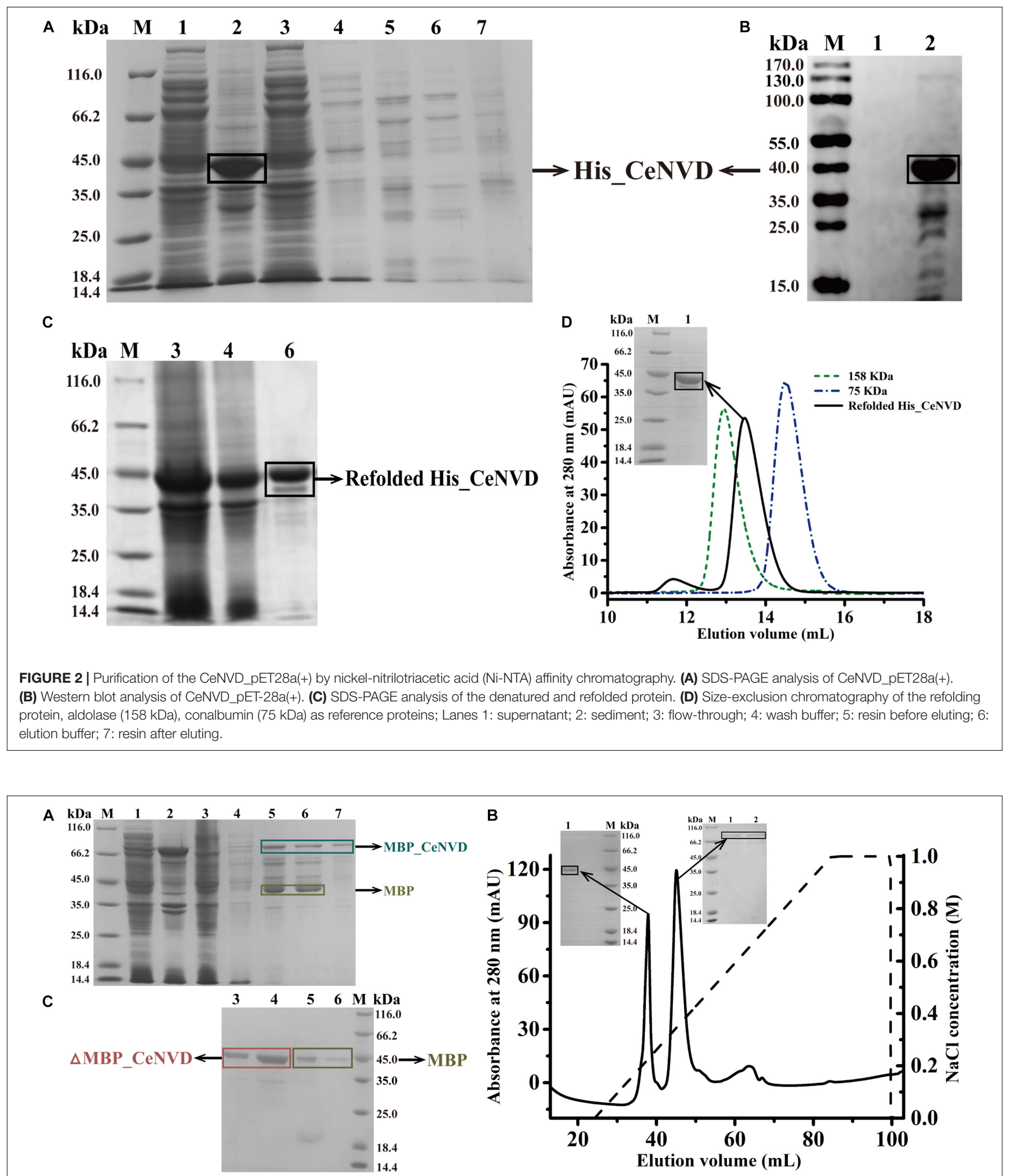

FIGURE 3 | (A) Purification of CeNVD_pMal-c2X by the MBP-trap affinity chromatography. (B) Purification of CeNVD_pMal-c2X by the anion-exchange.

(C) Purification of CeNVD by the MBP-trap without the MBP tag. Lanes 1: supernatant; 2: sediment; 3: flow-through; 4: wash buffer; 5: resin before eluting; 6: elution buffer; 7 : resin after eluting. 
TABLE 1 | Kinetic parameters of KsdD3 WT and mutants toward nine substrates.

\begin{tabular}{|c|c|c|c|c|}
\hline Purification step & Total protein $(\mathrm{mg})$ & Target protein (mg) & Purity (\%) & Yield (\%) \\
\hline Supernatant of MBP_CeNVD & 72.3 & 21.4 & 29.6 & 100 \\
\hline Eluate from Resource Q & 12.6 & 9.7 & 77.0 & 45.3 \\
\hline Flow through from the MBP-trap after digesting the MBP-tag & 4.5 & 4.1 & 91.1 & 19.2 \\
\hline Eluate from the Ni-NTA resin after refolding & 8.7 & 6.4 & 73.6 & 29.9 \\
\hline Eluate from Superdex200 & 3.6 & 3.2 & 88.9 & 15.0 \\
\hline
\end{tabular}

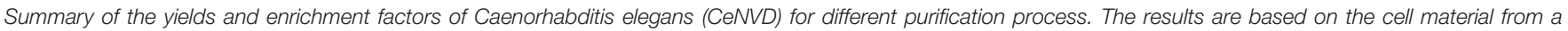
200-ml Escherichia coli culture.
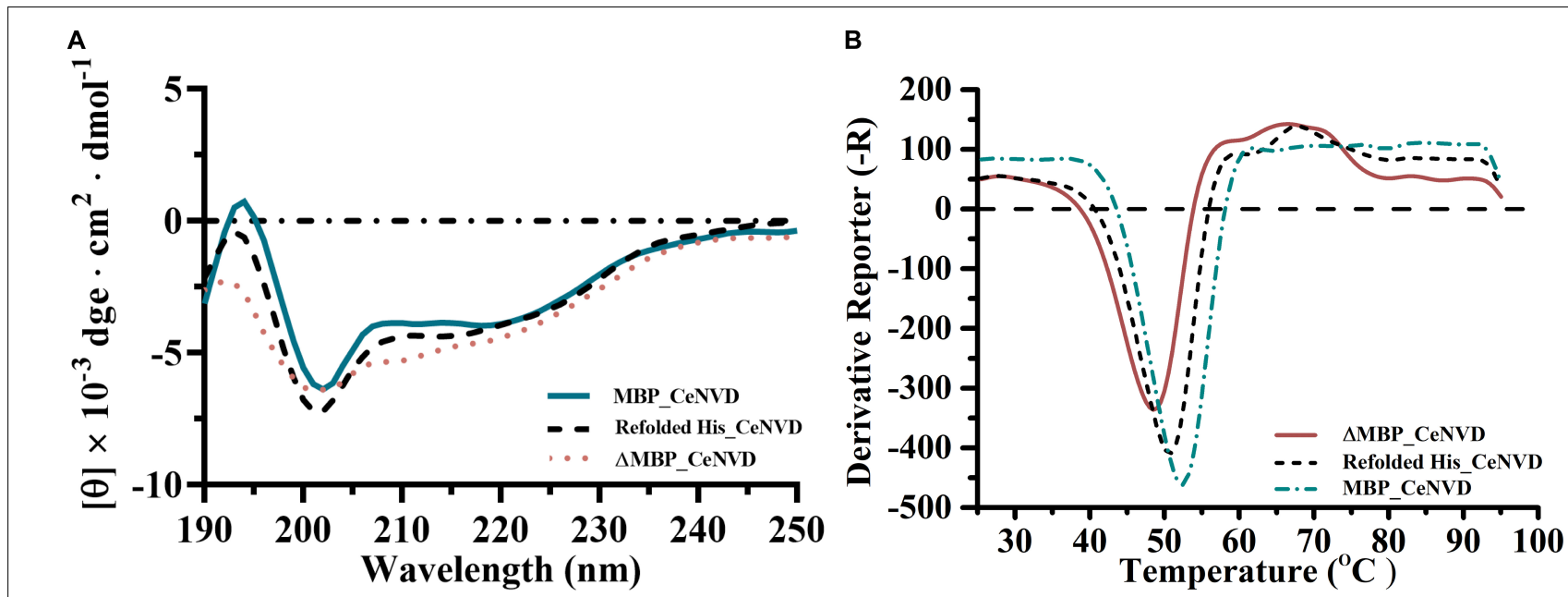

FIGURE 4 | (A) The CD spectra and (B) melting curves of CeNVD. The experiments were conducted in three replicates, and the data represent the means \pm standard deviations.

TABLE 2 | Secondary structure assignments (\%) of CeNVD determined by circular dichroism (CD) spectroscopy in the wavelength region from 190 to $250 \mathrm{~nm}$.

\begin{tabular}{lccccc}
\hline Protein & $\alpha$-Helix & \multicolumn{2}{c}{$\beta$-Strand } & \multirow{2}{*}{ Turn } & Other \\
\cline { 3 - 4 } & & & & \\
\cline { 3 - 4 } & & Antiparallel & Parallel & & \\
\hline Refolded His_CeNVD & 16.4 & 11.9 & 13.4 & 6.7 & 51.6 \\
MBP_CeNVD & 9.5 & 8.9 & 15.8 & 8.5 & 57.3 \\
MMBP_CeNVD & 22.5 & 17 & 10.1 & 4.6 & 45.8 \\
\hline
\end{tabular}

of CeNVD with $91.1 \%$ high purity was obtained in $200-\mathrm{ml}$ of cell culture. Therefore, the MBP was advantageous to the soluble expression and purification of CeNVD in E. coli, and the purification multiple-step purification method was necessary to obtain highly purified recombinant CeNVD.

\section{Characterization of the Refolded His_CeNVD}

His_CeNVD was expressed as inclusion bodies. Therefore, we added arginine and urea to refold the protein with an extra redox system to improve the refolding yield, such as reduced and oxidized glutathione (GSH and GSSG) (Chen et al., 2016). Here, we used a gradient descent of urea with $500 \mathrm{mM}$ arginine and a redox pair (GSH and GSSG) to facilitate the protein solubilizing and refolding. After dialysis, the refolded His_CeNVD was further purified by an Ni-NTA affinity chromatography (Figure 2C), and the fraction was further analyzed through a gel filtration chromatography, and a single peak was detected at $280 \mathrm{~nm}$. Consistent with the gel filtration chromatography analysis, SDS-PAGE indicated that the refolded His_CeNVD of higher purity (88.9\%) (Table 1) was obtained and a trimer state in solution (Figure 2D).

\section{Characterization of All CeNVD}

The secondary structure of the MBP_CeNVD, $\triangle \mathrm{MBP} \_$CeNVD, and refolded His_CeNVD were measured by CD spectroscopy (Figure 4A). The MBP_CeNVD showed a negative absorption peak centered around $202 \mathrm{~nm}$, and the percentage of $\alpha$-helix, $\beta$-strand, turn, and unordered regions were 9.5\%, $24.7 \%, 8.5 \%$, and $57.3 \%$, respectively. The CD spectrum of the $\triangle \mathrm{MBP} \_C e N V D$ and refolded protein demonstrated a visible increase in $\alpha$-helix $(22.5 \%, 16.4 \%)$ and a slight decrease in turn structures $(4.6 \%$, $6.7 \%$ ) and unstructured regions (45.8\% and 51.6\%) (Table 2).

Fluorescence-based thermal shift assay was used to determine the thermostability of the three types of CeNVD. As shown in Figure 4B, the $T_{m}$ value of the MBP_CeNVD was higher than the His_CeNVD and $\triangle \mathrm{MBP}-\mathrm{NVD}\left(52.5^{\circ} \mathrm{C}, 50^{\circ} \mathrm{C}\right.$, and $48^{\circ} \mathrm{C}$ ), which suggested that the $\mathrm{MBP}$ was profitable for the thermostability of CeNVD. 

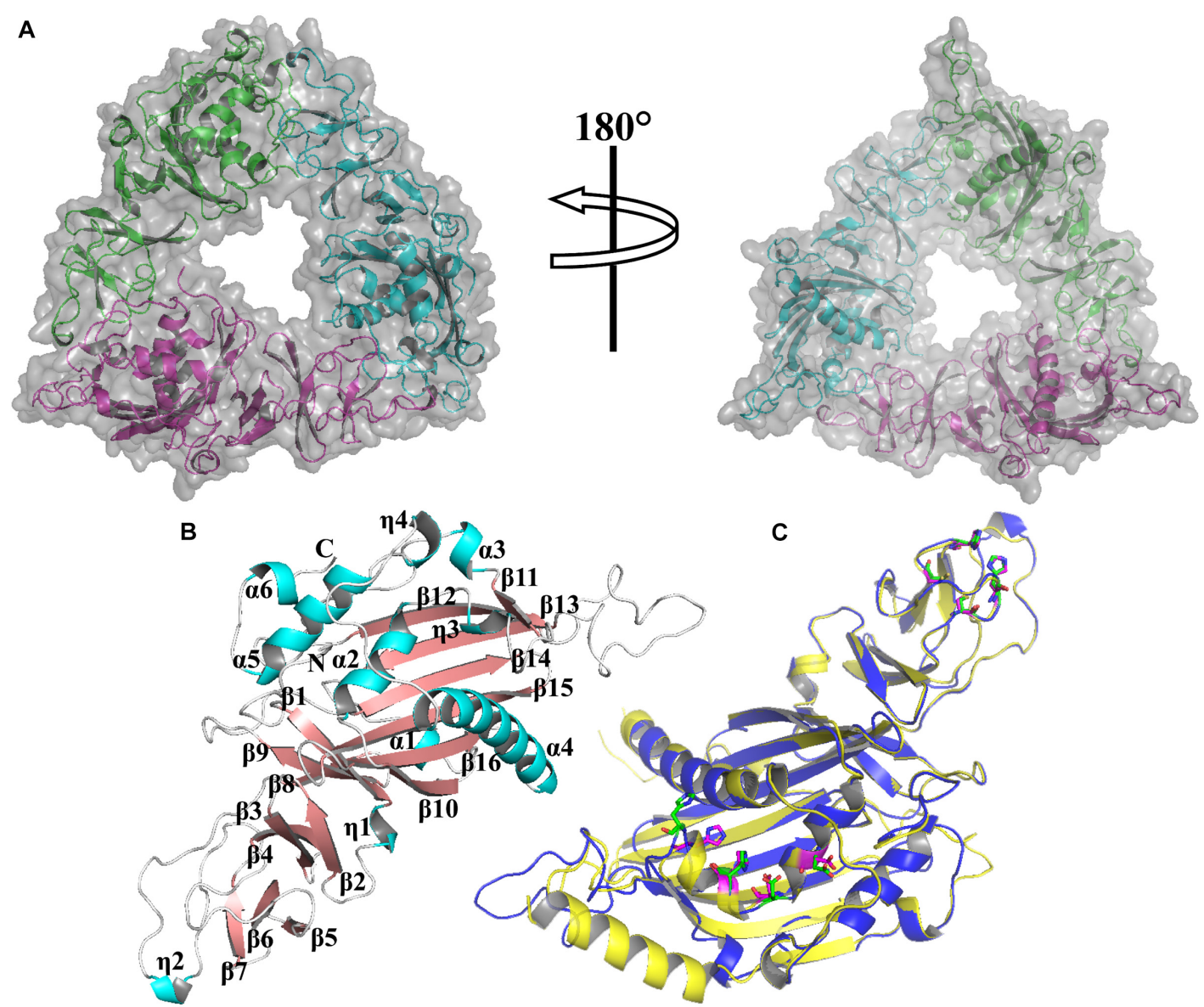

$\alpha 3$

C
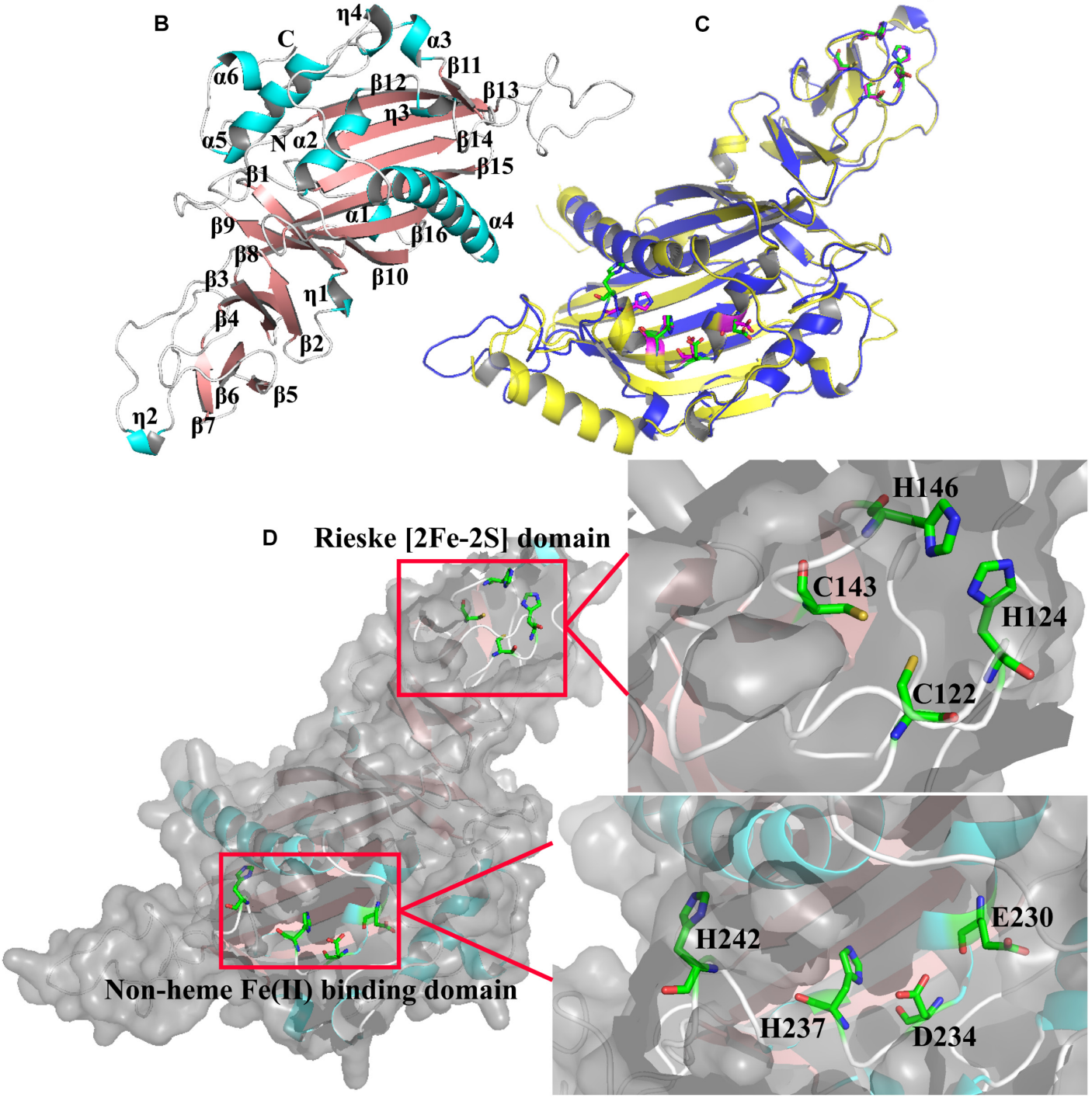

FIGURE 5 | (A) The structure model of CeNVD. (B) Monomer structure of CeNVD with the $\alpha$-helices (cyan) and $\beta$-strands (salmon) are labeled. (C) The superimposed subunits of CeNVD (blue) and the 3-ketosteroid $9 \alpha$-hydroxylases from Rhodococcus rhodochrous (yellow) with major domains; residues are shown as green and magenta sticks. (D) The stereo view of Rieske [2Fe-2S] domain and non-heme Fe(II)-binding domain. 


\section{Structural Analysis of CeNVD}

The structure of CeNVD contains six $\alpha$-helices $(\alpha 1-\alpha 6)$ and 16 antiparallel $\beta$-strands $(\beta 1-\beta 16)$ (Figures 5A,B), which are conserved in the NVD family enzymes. It contains an $N$-terminal Rieske [2Fe-2S] cluster (C122, H124, C143, H146) and followed by the catalytic domain harboring non-heme Fe (II) center (E230, D234, H237, H242). The Rieske cluster was located at strands $\beta 4, \beta 5$ and $\beta 6, \beta 7$. As for the Fe (II) center, E230 and D234 are located at $\alpha 2, \mathrm{H} 237$ is located at $\eta 3$, and H242 is located on a loop between $\eta 3$ and $\beta 11$ (Figure 5D; Penfield et al., 2014). Structural alignment revealed that the structure and residues in the major domains are remarkably similar to that of 3-ketosteroid $9 \alpha$-hydroxylases from $R$. rhodochrous (C67, H69, C86, H89) and (D174, D178, H181, H186) (Figure 5C; Mao et al., 2020b).

\section{CONCLUSION}

Our study provides a methodological approximation for the purification of soluble Rieske domain-containing oxygenase expressed in E. coli. We successfully expressed and purified the CeNVD protein in $E$. coli with the soluble formation of refolded His-NVD and MBP-NVD, showing that the MBP tag could increase the soluble expression of CeNVD, which is advantageous for further purification and improvement of thermostability.

\section{DATA AVAILABILITY STATEMENT}

The raw data supporting the conclusions of this article will be made available by the authors, without undue reservation.

\section{REFERENCES}

Chen, Y., Wang, Q., Zhang, C., Li, X., Gao, Q., Dong, C., et al. (2016). Improving the refolding efficiency for proinsulin aspart inclusion body with optimized buffer compositions. Protein Expr. Purif. 122, 1-7. doi: 10.1016/j.pep.2016.01. 015

Di Rosa, M., Malaguarnera, M., Nicoletti, F., and Malaguarnera, L. (2011). Vitamin D3: a helpful immuno-modulator. Immunology 134, 123-139. doi: 10.1111/j. 1365-2567.2011.03482.x

Dugas, D., and Brunel, J. M. (2006). Synthesis of 7-dehydrocholesterol through a palladium catalyzed selective homoannular conjugated diene formation. J. Mol. Catal. A Chem. 253, 119-122. doi: 10.1016/J.MOLCATA.2006. 03.014

Guex, N., and Peitsch, M. C. (1997). SWISS-MODEL and the Swiss-Pdb Viewer: an environment for comparative protein modeling. Electrophoresis 18, 2714-2723. doi: 10.1002/elps.1150181505

Holt, R. A., Subramanian, G. M., Halpern, A., Sutton, G. G., Charlab, R., Nusskern, D. R., et al. (2002). The genome sequence of the malaria mosquito Anopheles gambiae. Science 298, 129-149. doi: 10.1126/science.1076181

Huang, X., Warren, J. T., and Gilbert, L. I. (2008). New players in the regulation of ecdysone biosynthesis. J. Genet. Genomics 35, 1-10. doi: 10.1016/S16738527(08)60001-6

Iga, M., Blais, C., and Smagghe, G. (2013). Study on ecdysteroid levels and gene expression of enzymes related to ecdysteroid biosynthesis in the larval testis of Spodoptera littoralis. Arch. Insect Biochem. Physiol. 82, 14-28. doi: 10.1002/ ARCH.21068

Kanis, J. A. (1999). Vitamin D analogs: from renal bone disease to osteoporosis. Kidney Int. 56, S77-S81. doi: 10.1046/J.1523-1755.1999.07317.X

\section{AUTHOR CONTRIBUTIONS}

FL and H-MQ designed the research. SM, ZS, MW, and XW performed the experiments and analyzed the data. H-MQ, SM, and FL wrote the manuscript. All authors read and approved the final manuscript.

\section{FUNDING}

This work was supported by the National Key Research and Development Program of China, Synthetic Biology Research (Grant No. 2019YFA0905300), National Natural Science Foundation of China (31771911 and 21878233), and Tianjin Synthetic Biotechnology Innovation Capacity Improvement Project (TSBICIP-KJGG-001-09).

\section{ACKNOWLEDGMENTS}

This manuscript has been released as a pre-print at https:// www.researchsquare.com/article/rs-41569/v1, doi: 10.21203/rs.3. rs- $41569 / \mathrm{v} 1$.

\section{SUPPLEMENTARY MATERIAL}

The Supplementary Material for this article can be found online at: https://www.frontiersin.org/articles/10.3389/fbioe. 2020.593041/full\#supplementary-material

Lang, M., Murat, S., Clark, A. G., Gouppil, G., Blais, C., Matzkin, L. M., et al. (2012). Mutations in the neverland gene turned Drosophila pachea into an obligate specialist species. Science 337, 1658-1661. doi: 10.1126/science.1224829

Lee, J., Simurdiak, M., and Zhao, H. (2005). Reconstitution and characterization of aminopyrrolnitrin oxygenase, a Rieske N-oxygenase that catalyzes unusual arylamine oxidation. J. Biol. Chem. 280, 36719-36727. doi: 10.1074/JBC. M505334200

Mao, S., Cheng, X., Zhu, Z., Chen, Y., Li, C., Zhu, M., et al. (2020a). Engineering a thermostable version of $\mathrm{D}$-allulose 3-epimerase from Rhodopirellula baltica via site-directed mutagenesis based on B-factors analysis. Enzyme Microb. Technol. 132:109411. doi: 10.1016/j.enzmictec.2019.10 9441

Mao, S., Song, Z., Wu, M., Wang, X., Lu, F., and Qin, H. M. (2020b). Expression, Purification, Refolding and Characterization of a Neverland Protein from Caenorhabditis elegans. Available online at: https://www.researchsquare.com/ article/rs-41569/v1 (accessed July 16, 2020).

Mao, S., Wang, J., Liu, F., Zhu, Z., Gao, D., Guo, Q., et al. (2018). Engineering of 3-ketosteroid- $\Delta 1$-dehydrogenase based site-directed saturation mutagenesis for efficient biotransformation of steroidal substrates. Microb. Cell Fact. 17:141. doi: 10.1186/s12934-018-0981-0

Micsonai, A., Wien, F., Bulyaki, E., Kun, J., Moussong, E., Lee, Y. H., et al. (2018). BeStSel: a web server for accurate protein secondary structure prediction and fold recognition from the circular dichroism spectra. Nucleic Acids Res. 46, W315-W322. doi: 10.1093/nar/gky497

Micsonai, A., Wien, F., Kernya, L., Lee, Y. H., Goto, Y., Refregiers, M., et al. (2015). Accurate secondary structure prediction and fold recognition for circular dichroism spectroscopy. Proc. Natl. Acad. Sci. U.S.A. 112, E3095-E3103. doi: $10.1073 /$ pnas. 1500851112 
Penfield, J. S., Worrall, L. J., Strynadka, N. C., and Eltis, L. D. (2014). Substrate specificities and conformational flexibility of 3-ketosteroid $9 \alpha$-hydroxylases. J. Biol. Chem. 289, 25523-25536. doi: 10.1074/jbc.M114.575886

Petrusma, M., Hessels, G., Dijkhuizen, L., and Van Der Geize, R. (2011). Multiplicity of 3-ketosteroid-9 $\alpha$-hydroxylase enzymes in Rhodococcus rhodochrous DSM43269 for specific degradation of different classes of steroids. J. Bacteriol. 193, 3931-3940. doi: 10.1128/JB.00274-11

Qin, H. M., Wang, J., Guo, Q., Li, S., Xu, P., Zhu, Z., et al. (2017a). Refolding of a novel cholesterol oxidase from Pimelobacter simplex reveals dehydrogenation activity. Protein Expr. Purif. 139, 1-7. doi: 10.1016/J.PEP.2017.07.008

Qin, H. M., Zhu, Z., Ma, Z., Xu, P., Guo, Q., Li, S., et al. (2017b). Rational design of cholesterol oxidase for efficient bioresolution of cholestane skeleton substrates. Sci. Rep. 7:16375. doi: 10.1038/S41598-017-16768-6

Romero, P., Wagg, J., Green, M. L., Kaiser, D., Krummenacker, M., and Karp, P. D. (2005). Computational prediction of human metabolic pathways from the complete human genome. Genome Biol. 6:R2. doi: 10.1186/gb-2004-6-1-r2

Rottiers, V., Motola, D. L., Gerisch, B., Cummins, C. L., Nishiwaki, K., Mangelsdorf, D. J., et al. (2006). Hormonal control of C. elegans dauer formation and life span by a Rieske-like oxygenase. Dev. Cell 10, 473-482. doi: 10.1016/J. DEVCEL.2006.02.008

Sun, D., Gao, D., Liu, X., Zhu, M., Li, C., Chen, Y., et al. (2019). Redesign and engineering of a dioxygenase targeting biocatalytic synthesis of 5-hydroxyl leucine. Catal. Sci. Technol. 9, 1825-1834. doi: 10.1039/C9CY00110G

Sun, D., Gao, D., Xu, P., Guo, Q., Zhu, Z., Cheng, X., et al. (2018). A novel L-leucine 5-hydroxylase from Nostoc piscinale unravels unexpected sulfoxidation activity toward L-methionine. Protein Expr. Purif. 149, 1-6. doi: 10.1016/J.PEP.2018. 04.009

Thummel, C. S., and Chory, J. (2002). Steroid signaling in plants and insectscommon themes, different pathways. Genes Dev. 16, 3113-3129. doi: 10.1101/ gad.1042102

Tripkovic, L., Lambert, H., Hart, K., Smith, C. P., Bucca, G., Penson, S., et al. (2012). Comparison of vitamin D2 and vitamin D3 supplementation in raising serum 25-hydroxyvitamin D status: a systematic review and meta-analysis. Am. J. Clin. Nutr. 95, 1357-1364. doi: 10.3945/ajcn.111.031070

Wan, D., Yang, X., Wang, Y., Zhu, H., Wang, T., Liu, Z., et al. (2016). Catalpol stimulates VEGF production via the JAK2/STAT3 pathway to improve angiogenesis in rats' stroke model. J. Ethnopharmacol. 191, 169-179. doi: 10. 1016/J.JEP.2016.06.030

Warren, J. T., Petryk, A., Marqués, G., Jarcho, M., Parvy, J. P., Dauphin-Villemant, C., et al. (2002). Molecular and biochemical characterization of two P450 enzymes in the ecdysteroidogenic pathway of Drosophila melanogaster. Proc. Natl. Acad. Sci. U.S.A. 99, 11043-11048. doi: 10.1073/PNAS.162375799
Wollam, J., Magomedova, L., Magner, D. B., Shen, Y., Rottiers, V., Motola, D. L., et al. (2011). The Rieske oxygenase DAF-36 functions as a cholesterol 7-desaturase in steroidogenic pathways governing longevity. Aging Cell 10, 879-884. doi: 10.1111/J.1474-9726.2011.00733.X

Yamanaka, N., Hua, Y. J., Mizoguchi, A., Watanabe, K., Niwa, R., Tanaka, Y., et al. (2005). Identification of a novel prothoracicostatic hormone and its receptor in the silkworm Bombyx mori. J. Biol. Chem. 280, 14684-14690. doi: 10.1074/JBC. M500308200

Yoshiyama, T., Namiki, T., Mita, K., Kataoka, H., and Niwa, R. (2006). Neverland is an evolutionally conserved Rieske-domain protein that is essential for ecdysone synthesis and insect growth. Development 133, 2565-2574. doi: 10.1242/DEV. 02428

Yoshiyama-Yanagawa, T., Enya, S., Shimada-Niwa, Y., Yaguchi, S., Haramoto, Y., Matsuya, T., et al. (2011). The conserved Rieske oxygenase DAF-36/Neverland is a novel cholesterol-metabolizing enzyme. J. Biol. Chem. 286, 25756-25762. doi: 10.1074/JBC.M111.244384

Zhan, S., Merlin, C., Boore, J. L., and Reppert, S. M. (2011). The monarch butterfly genome yields insights into long-distance migration. Cell 147, 1171-1185. doi: 10.1016/J.CELL.2011.09.052

Zhu, Z., Gao, D., Li, C., Chen, Y., Zhu, M., Liu, X., et al. (2019a). Redesign of a novel D-allulose 3-epimerase from Staphylococcus aureus for thermostability and efficient biocatalytic production of D-allulose. Microb. Cell Fact. 18:59. doi: 10.1186/S12934-019-1107-Z

Zhu, Z., Li, C., Cheng, X., Chen, Y., Zhu, M., Liu, X., et al. (2019b). Soluble expression, purification and biochemical characterization of a C-7 cholesterol dehydrogenase from Drosophila melanogaster. Steroids 152:108495. doi: 10. 1016/J.STEROIDS.2019.108495

Zhu, Z., Li, C., Liu, X., Gao, D., Wang, X., Tanokura, M., et al. (2019c). Biochemical characterization and biocatalytic application of a novel D-tagatose 3-epimerase from Sinorhizobium sp. RSC Adv. 9, 2919-2927. doi: 10.1039/c8ra1 $0029 b$

Conflict of Interest: The authors declare that the research was conducted in the absence of any commercial or financial relationships that could be construed as a potential conflict of interest.

Copyright (c) 2020 Mao, Song, Wu, Wang, Lu and Qin. This is an open-access article distributed under the terms of the Creative Commons Attribution License (CC BY). The use, distribution or reproduction in other forums is permitted, provided the original author(s) and the copyright owner(s) are credited and that the original publication in this journal is cited, in accordance with accepted academic practice. No use, distribution or reproduction is permitted which does not comply with these terms. 